\title{
Structure and evolution of a dust star in the Oppenheimer-Snyder model
}

\author{
Zahid Zakir $^{1}$
}

\begin{abstract}
In 1939 Oppenheimer and Snyder (OS) have found an exact solution of the Einstein equations for a collapsing homogeneous dust star at the parabolic velocity of dust particles by transforming the Tolman solution in the comoving coordinates to a solution in the Schwarzschild coordinates $r, t$ and matching on the surface of the star with the exterior Schwarzschild solution. However, despite the regularly citation of the OS paper, the meaning and significance of their solution have so far remained unappreciated and poorly understood, in addition their method has been forgotten. In the present paper it is shown that the OS method allows one to describe correctly from the astrophysical point of view the structure and evolution of the dust star as a whole on hypersurfaces of simultaneity $\mathrm{t}=$ const. A detailed derivation of the parabolic OS solution and solutions for hyperbolic and elliptic velocities is given. The plots of the proper time rate and particle trajectories $r(t, R)$ in different layers are presented, visualizing the structure of the dust star. At large $t$, not only the surface quickly freezes outside the gravitational radius, asymptotically approaching it, but the particles in the internal layers also freeze at certain distances from the center, and their worldlines approach their own asymptotes, rapidly becoming almost parallel to the worldlines of particles at the center and on the surface. This shows that in the OS model the frozen star picture refers not only to the surface, but also to the structure of the collapsed dust star as a whole. Thus, at any finite moment of cosmological time the collapsed OS dust star appears as not a black hole, but as a frozar, an object by practically totally frozen internal structure.
\end{abstract}

PACS: 04.20.Dg; 04.70.-s; 97.60.-s, 98.54.-h

Keywords: relativistic stars, gravitational collapse, black holes, quark stars

\section{Content}

Introduction

1. Trajectories in the Schwarzschild field and Tolman's solution for the dust ball ............19

1.1. Trajectories of particles in the Schwarzschild field .......................................................19

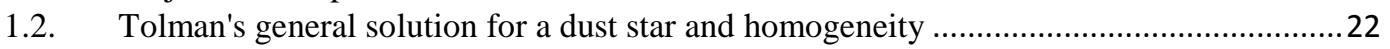

1.3. Two additional consequences of homogeneity ........................................................23

2. A homogeneous dust star on a hypersurface of simultaneity .....................................24

2.1. The metric and trajectories in the exterior region .....................................................24

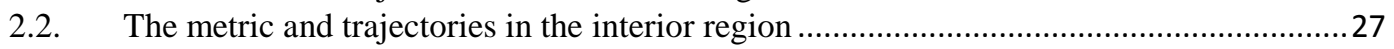

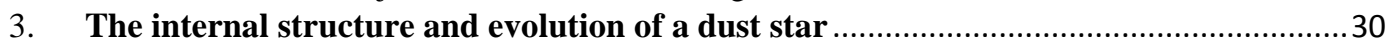

3.1. Trajectories of particles in layers and their asymptotics ............................................. 30

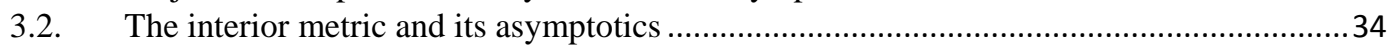

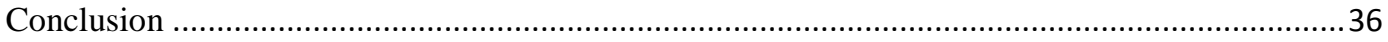

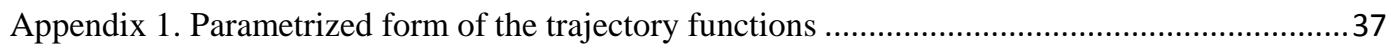

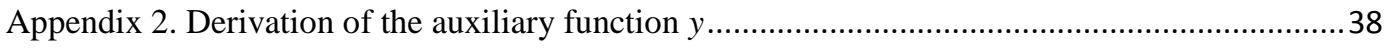

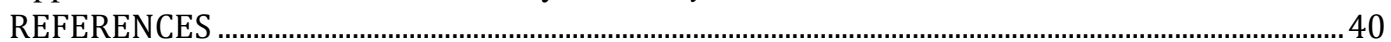

${ }^{1}$ Centre for Theoretical Physics and Astrophysics, Tashkent, Uzbekistan, zahidzakir@theor-phys.org 


\section{Introduction}

In determining the structure of collapsing stars with a mass $M>3 M_{\circ}$, when their surface is close to the gravitational radius $r_{g}=2 G M$ ( $G$ is the gravitational constant) and gravity dominates, there are still many ambiguities. In the simplest model - a spherical star with homogeneous dust matter and with a diagonal metric - the problem turned out to be exactly solvable (Friedmann, 1922; Lemaître, 1933; Tolman R., 1934) and, therefore, the internal structure of the such «ideal star» at each moment should have been well studied.

However, these exact solutions were found under the condition of homogeneity in the comoving to the dust particles local frames which are mutually moving and, therefore, do not have a common hypersurface of simultaneity. At the same time, the determination of the structure of an extended object «at a given moment» means taking the positions of all its particles simultaneously, i.e. on a common hypersurface of simultaneity. In addition, the time of this hypersurface must coincide on the surface of the star with the world time of the exterior region. For these reasons, the existence of the solutions in the comoving frames is not enough for determining the structure of the star as a whole.

In the case of a homogeneous dust star with a parabolic velocity of dust particles this problem was solved by OS (Oppenheimer \& Snyder, 1939) by transforming the known exact solution in the comoving coordinates (Tolman R., 1934) into the Schwarzschild coordinates $(t, r, \theta, \varphi)$ (Schwarzschild, 1916) on the global hypersurface of simultaneity $t=$ const . Unlike other attempts (Datt, 1938; Klein, 1961; Weinberg, 1972), where the diagonality of the such transformed metric is achieved by a local transformation of the coordinate time, in the OS method this is achieved by maintaining the world time of the exterior region. This ensures both to match correctly on the surface with the exterior metric, and to describe the star at every moment of the world time as a totality of simultaneous events. Since then, the OS solution has been used as a standard model for normalizing and testing more complicated models of relativistic stars.

The aim of the present paper is to develop the OS model by its more detailed and consistent formulation, the determination of asymptotes at $t \gg r_{g}$ not only for the metric, but also for the trajectories of the star's particles with the visualization of these trajectories, and the derivation of exact interior solutions in the Schwarzschild coordinates for the elliptic and hyperbolic velocities.

In Section 1 the main relations for particle trajectories in the Schwarzschild field and the Tolman solution for the dust ball are discussed. In Section 2, the exact solutions by the OS method for all three velocity regimes are derived. In Section 3, the structure and evolution of the dust star are studied and the plots of the worldlines are presented, clearly showing the structure of the star at a given time $t$ and at the asymptotes $t \rightarrow \infty$. In the Appendices a parametrized representation of the trajectory equations and the derivation of the solutions for the OS auxiliary functions $y(R, r)$ are presented. 


\section{Trajectories in the Schwarzschild field and Tolman's solution for the dust ball}

\subsection{Trajectories of particles in the Schwarzschild field}

The space-time interval outside and on the surface of the spherical star $r \geq r_{b}$ (where $r_{b}$ is the circumferential radius of the surface) in the static coordinates is given by the Schwarzschild solution:

$$
d s^{2}=\left(1-r_{g} / r\right) d t^{2}-\left(1-r_{g} / r\right)^{-1} d r^{2}-r^{2} d^{2} \Omega,
$$

where $d^{2} \Omega=d \theta^{2}+\sin ^{2} \theta d \varphi^{2}$. At the radial falling of test particles in this field their local velocities have the form:

$$
v^{2}=\frac{1}{\left(1-r_{g} / r\right)^{2}} \frac{d r^{2}}{d t^{2}} .
$$

The energy conservation condition for freely falling particles in this static field gives:

$$
\frac{1-r_{g} / r}{1-v^{2}(r)}=1+f(R)
$$

where the form of $f(R)$ is fixed at the initial moment at $r=R$.

From these basic expressions, then we find the trajectory functions for test particles outside the star for three kinds of velocities - parabolic ( $f=0)$, hyperbolic $\left(f=r_{g} / R\right)$, and elliptic $\left(f=-r_{g} / R\right)$. They are distinguished by the fact that they correspond to a constant curvature space and an initially homogeneous set of test particles remains homogeneous in the future for the same intervals of the local proper time. The hypersurfaces of «one-age» for this reason are also the hypersurfaces of homogeneity.

\section{a. Parabolic velocities.}

The parabolic velocity case with $f=0$ corresponds to the velocity which would be achieved at a free fall from the rest at $r \rightarrow \infty$. The energy conservation condition for particles (3) gives in this case

$$
\frac{1-r_{g} / r}{1-v^{2}}=1, \quad v=-r_{g}^{1 / 2} / r^{1 / 2}
$$

For the proper time interval of the particles it follows from (1), (2) and (3):

$$
d \tau^{2}=\frac{d r^{2}}{1-r_{g} / r}\left(\frac{1}{v^{2}}-1\right)=\frac{r}{r_{g}} d r^{2},
$$

By defining the initial positions of particles at $\tau=0$ as $r=R$, we obtain from (5):

$$
\tau(R, r)=\frac{2}{3 r_{g}^{1 / 2}}\left(R^{3 / 2}-r^{3 / 2}\right), \quad r(\tau, R)=\left(R^{3 / 2}-\frac{3}{2} r_{g}^{1 / 2} \tau\right)^{2 / 3} .
$$

For the derivatives $\dot{r}=\partial r / \partial \tau$ and $r^{\prime}=\partial r / \partial R$ we have from (5)-(6):

$$
\dot{r}=-\frac{r_{g}^{1 / 2}}{r^{1 / 2}}, \quad r^{\prime}=\frac{R^{1 / 2}}{r^{1 / 2}} .
$$

For the world time interval $t$ it follows from (2) and (3):

$$
t-t_{0}(R)=-\frac{1}{r_{g}^{1 / 2}} \int_{R}^{r(t)} \frac{d r r^{3 / 2}}{r-r_{g}}=\frac{1}{r_{g}^{1 / 2}} \int_{r(t)}^{R} \frac{d r r^{3 / 2}}{r-r_{g}},
$$


where $t_{0}(R)$ is a value of $t$ corresponding to the moment $\tau=0$ for the layer $R$. The integration of (8) gives the trajectory function $r(t, R)$ in the implicit form:

$$
t-t_{0}(R)=\frac{2}{3 r_{g}^{1 / 2}}\left(R^{3 / 2}-r^{3 / 2}\right)+2 r_{g}^{1 / 2}\left(R^{1 / 2}-r^{1 / 2}\right)+r_{g} \ln \left(\frac{r^{1 / 2}+r_{g}^{1 / 2}}{r^{1 / 2}-r_{g}^{1 / 2}} \frac{R^{1 / 2}-r_{g}^{1 / 2}}{R^{1 / 2}+r_{g}^{1 / 2}}\right) .
$$

From (9), using (7), we find the derivative $\dot{t}$ :

$$
\dot{t}=\frac{r}{r-r_{g}} \text {. }
$$

For the calculating $t^{\prime}$, an explicit form of $t_{0}(R)$ is required and in Section 2.2 it will be found from the metrics diagonality condition in the comoving frame of reference.

\section{b. Hyperbolic and elliptic velocities.}

The hyperbolic velocities correspond to a finite velocity $v_{\infty}^{2}>0$ at $r \rightarrow \infty$ and in (3) they correspond to the choice $f(R)=r_{g} / R>0$. The elliptic velocities correspond to falling from the rest $v(R)=0$ at a finite distance $r=R<\infty$ at the initial moment $\tau=0$ and the energy conservation condition (3) gives in this case $f(R)=-r_{g} / R<0$. Then both cases will be considered together and the sign $(+)$ will be for the hyperbolic, and the sign $(-)$ - for the elliptic velocities.

Thus, from (3) we obtain:

$$
\begin{gathered}
\frac{1-r_{g} / r}{1-v^{2}}=1 \pm \frac{r_{g}}{R}, \\
v^{2}=A \cdot \frac{R \pm r}{r}, \quad A=\frac{r_{g}}{R \pm r_{g}} .
\end{gathered}
$$

From (1) - (2) and the expressions for the velocity (12) we find for the proper time interval of the particle:

$$
d \tau^{2}=\frac{d r^{2}}{1-r_{g} / r}\left(\frac{1}{v^{2}}-1\right)=\frac{R}{r_{g}} \cdot \frac{r d r^{2}}{R \pm r},
$$

from which it follows the relationship between $\tau$ and $r$ along any trajectory $\tau(R, r)$ :

$$
\begin{gathered}
\tau=\frac{R^{1 / 2}}{r_{g}^{1 / 2}} \int_{r(\tau)}^{R} \frac{d r r^{1 / 2}}{(R \pm r)^{1 / 2}}, \\
\tau=\frac{R^{1 / 2}}{r_{g}^{1 / 2}}\left[2^{1 / 2} R-[r(R+r)]^{1 / 2}\right]+\frac{R^{3 / 2}}{r_{g}^{1 / 2}} \ln \left(\frac{r^{1 / 2}+(R+r)^{1 / 2}}{R^{1 / 2}\left(1+2^{1 / 2}\right)}\right), \quad f>0 \\
\tau=\frac{R^{1 / 2}}{r_{g}^{1 / 2}}[r(R-r)]^{1 / 2}+\frac{R^{3 / 2}}{r_{g}^{1 / 2}} \arccos \left(r^{1 / 2} / R^{1 / 2}\right), \quad f<0 .
\end{gathered}
$$

The derivative $\dot{r}$ follows from (13):

$$
\dot{r}=-\frac{r_{g}^{1 / 2}}{r^{1 / 2}}(1 \pm r / R)^{1 / 2},
$$

while $r^{\prime}=\partial r / \partial R$ we find from (15) and (16) by using the condition $\tau^{\prime}=0$ : 


$$
\begin{aligned}
& r^{\prime}=-\frac{3}{2}-\frac{r}{2 R}+\frac{3}{2}\left[2^{1 / 2}+\ln \frac{(1+r / R)^{1 / 2}+(r / R)^{1 / 2}}{1+2^{1 / 2}}\right](1+r / R)^{1 / 2}, f>0, \\
& r^{\prime}=\frac{3}{2}-\frac{r}{2 R}+\frac{3}{2}\left(\frac{R}{r}-1\right)^{1 / 2} \arccos \left(r^{1 / 2} / R^{1 / 2}\right), f<0 .
\end{aligned}
$$

Notice that the logarithmic term in (18) can be written across $\operatorname{arccosh}\left[(r / R)^{1 / 2}\right]$.

For the world time interval $t$, it follows from (2) and (12) that:

$$
t=\frac{1}{A^{1 / 2}} \int_{r(t)}^{R} \frac{d r r^{3 / 2}}{\left(r-r_{g}\right)(R \pm r)^{1 / 2}},
$$

and the calculation of the integral gives a relationship between $t$ and $r$ along the trajectories $t(R, r)$ :

$$
\begin{aligned}
& t-t_{0}(R)=2 r_{g} \ln \frac{\left[r\left(R+r_{g}\right)\right]^{1 / 2}+\left[r_{g}(R+r)\right]^{1 / 2}}{\left[R\left(r-r_{g}\right)\right]^{1 / 2}}+ \\
& +\left[\left(R-2 r_{g}\right) \ln \frac{r^{1 / 2}+(R+r)^{1 / 2}}{R^{1 / 2}}-[r(r+R)]^{1 / 2}\right]\left(1+R / r_{g}\right)^{1 / 2}, f>0 \\
& t-t_{0}(R)=2 r_{g} \ln \frac{\left[r\left(R-r_{g}\right)\right]^{1 / 2}+\left[r_{g}(R-r)\right]^{1 / 2}}{\left[R\left(r-r_{g}\right)\right]^{1 / 2}}+ \\
& +\left(\left(R+2 r_{g}\right) \arccos \left(r^{1 / 2} / R^{1 / 2}\right)+[r(R-r)]^{1 / 2}\right)\left(\frac{R}{r_{g}}-1\right)^{1 / 2}, f<0
\end{aligned}
$$

From these equations for the trajectory, taking into account (17) - (19), we find $\dot{t}$ :

$$
\dot{t}=-\frac{\left[r\left(R \pm r_{g}\right)\right]^{1 / 2}}{\left[r_{g}(R \pm r)\right]^{1 / 2}} \frac{\dot{r}}{1-r_{g} / r}=\frac{\left(1 \pm r_{g} / R\right)^{1 / 2}}{1-r_{g} / r} .
$$

в. A relation of the proper time to the world time and its asymptotes.

The times $\tau$ and $t$ are two parametrizations of the same events along the same worldline of the particle $r=r(\tau, R)=r(t, R)$ and, therefore, these two times are mutually related, and irreversible gravitational dilation of $\tau$ w.r.t. $t$ is the experimental fact (see (Okun, Selivanov, \& Telegdi, 2000)).

The relation between $\tau$ and $t$ in the implicit form can be obtained from (9) and (16) by calculating $t(r, R)$ and $\tau(r, R)$ at the same values of $r$ and $R$. At the same time, while $r>r_{g}$ both $t(r, R)$ and $\tau(r, R)$ describe the same event on the same worldline (at fixed angles $\varphi, \theta)$. Therefore, if the worldline $t(r, R)$ asymptotically approaches $r_{g}$ by never crossing it, then the worldline $\tau(r, R)$ also asymptotically approaches $r_{g}$ and also does not cross it. The proper time at $t \rightarrow \infty$ and $r \rightarrow r_{g}$ only asymptotically freezes, tending to the moment of freezing $\tau_{g}$, which we find from (6), (15), and (16):

$$
\tau_{g}=\frac{2}{3 r_{g}^{1 / 2}}\left(R^{3 / 2}-r_{g}^{3 / 2}\right), \quad f=0,
$$




$$
\begin{aligned}
& \tau_{g}=\frac{R^{1 / 2}}{r_{g}^{1 / 2}}\left[2^{1 / 2} R-\left[r_{g}\left(R+r_{g}\right)\right]^{1 / 2}\right]+\frac{R^{3 / 2}}{r_{g}^{1 / 2}} \ln \left(\frac{r_{g}^{1 / 2}+\left(R+r_{g}\right)^{1 / 2}}{R^{1 / 2}\left(1+2^{1 / 2}\right)}\right), f>0, \\
& \tau_{g}=\left[r_{g}\left(R-r_{g}\right)\right]^{1 / 2}+\frac{R^{3 / 2}}{r_{g}^{1 / 2}} \arccos \left(r_{g}^{1 / 2} / R^{1 / 2}\right), \quad f<0 .
\end{aligned}
$$

The plots of the dependences $\tau(t, R)$ for all three velocities are shown below in Fig. 2, where the positions of the asymptotes $\tau_{g}$ are shown also.

\subsection{Tolman's general solution for a dust star and homogeneity}

Inside the dust star $\left(R \leq R_{b}\right)$, the line element in the comoving local frames of reference can be written in the form:

$$
d s^{2}=c^{2} d \tau^{2}-e^{\lambda(\tau, R)} d R^{2}-r^{2}(\tau, R) d \Omega^{2},
$$

where $2 \pi r(\tau, R)$ is the length of a circle around the star.

Tolman's general solution for the Einstein equations with the such metric (Tolman R., 1934) (in the notation of (Landau \& Lifshits, 1994)) has the form:

$$
e^{\lambda}=r^{\prime 2} /(1+f), \quad \dot{r}^{2}=f+F / r, \quad 8 \pi G \rho=\frac{F^{\prime}}{r^{\prime} r^{2}} .
$$

where $1+f>0, \rho$ is the energy density of the dust matter. It contains two unknown functions $f(R)$ and $F(R)$, and thus in order to find the exact solutions it is necessary to specify the physical conditions.

For our purposes, homogeneity is the first such condition. In local comoving reference frames, where Tolman's solution is valid, the homogeneity condition means that for the same values of proper time (for the «one-age» events) $\rho$ does not depend on the spatial coordinates:

$$
\rho=\rho(\tau)
$$

This can be achieved by the choice of a homogeneous distribution at the initial moment $\tau=0$ and by choosing one of the three special velocities that preserve the homogeneity during the time evolution, since they correspond to the constant curvature spaces.

The integration of (28) then gives:

$$
\tau= \pm \int \frac{d r}{(f+F / r)^{1 / 2}} .
$$

In addition in the exterior region $F=r_{g}$.

For the parabolic motion ( $f=0)$ we obtain from (28) - (30):

$$
\tau=\frac{2}{3 F^{1 / 2}}\left(R^{3 / 2}-r^{3 / 2}\right), \quad r=\left(R^{3 / 2}-\frac{3 F^{1 / 2}}{2} \tau\right)^{2 / 3}, \dot{r}=-\frac{F^{1 / 2}}{r^{1 / 2}} .
$$

In the elliptic case, $f$ is determined from the vanishing of the velocity at $r=R$ :

$$
\dot{r}^{2}(R)=f+\frac{F}{R}=0, \quad f=-\frac{F}{R}<0 .
$$

In the hyperbolic case, it should be the same value of $f$, but with the opposite sign: $f=F / R$.

Thus, in the hyperbolic and elliptic cases we have for $\dot{r}^{2}$ : 


$$
\dot{r}=-\frac{F^{1 / 2}}{r^{1 / 2}}\left(1 \pm \frac{r}{R}\right)^{1 / 2}
$$

The integration of (33) is analogous to (14) - (16) and gives:

$$
\begin{aligned}
& \tau=\frac{R^{1 / 2}}{F^{1 / 2}}\left[2^{1 / 2} R-[r(R+r)]^{1 / 2}\right]+\frac{R^{3 / 2}}{F^{1 / 2}} \ln \left(\frac{r^{1 / 2}+(R+r)^{1 / 2}}{R^{1 / 2}\left(1+2^{1 / 2}\right)}\right), \quad f>0 \\
& \tau=\frac{R^{1 / 2}}{F^{1 / 2}}\left[[r(R-r)]^{1 / 2}+R \arccos \left(r^{1 / 2} / R^{1 / 2}\right)\right], \quad f<0 .
\end{aligned}
$$

\subsection{Two additional consequences of homogeneity}

Thus, the condition to be comoving leads to the diagonal metric (27) with which the Einstein equations have an exact solution (28) by two unknown functions $f$ and $F$, and then the homogeneity condition and the choice of one of the three special velocities allow to determine $f$.

At the first look it seems that there remains the arbitrariness in the determination of the second of the unknown functions $F$. Below we show that in reality there is no such arbitrariness, and by the above accepted refinements of physical conditions it is fully determined not only $f$, but $F$ also. In the OS paper this was shown for parabolic velocities, and here we show that the form of $F$ is the same for all three types of special velocities.

For this purpose, from (28) and the homogeneity condition (29), we obtain the following relations $(\kappa=8 \pi G / 3)$ :

$$
\begin{gathered}
F^{\prime}=3 \kappa \rho(\tau) r^{2} r^{\prime}=\kappa \rho(\tau)\left(r^{3}\right)^{\prime}, \\
F=\kappa \rho(\tau) r^{3}=\kappa \rho(0) R^{3}=\kappa \rho(0) R_{b}^{3} \frac{R^{3}}{R_{b}^{3}} .
\end{gathered}
$$

Further, taking into account $r_{g}=2 G M=\kappa \rho(0) R_{b}^{3}$, we arrive at the expressions:

$$
F(R)=r_{g} \frac{R^{3}}{R_{b}^{3}} \equiv r_{g, R}, \quad \rho(\tau)=\rho(0) \frac{R^{3}}{r^{3}} .
$$

Thus, the first additional consequence of homogeneity is that the form of $F$ inside the star is uniquely determined from (28) and (29), and the form of $F$ is the same for all three special velocities, since in deriving of (38), in contrast to OS, the concrete form of $f$ was not used.

Knowing $f$ and $F$, now we can calculate from (31) and (33) not only $\dot{r}$, but also $r^{\prime}$, if we again use (36) - (38). At first, from (36) - (37) we obtain:

$$
F^{\prime}=\kappa \rho(\tau) r^{2} r^{\prime}=\kappa \rho(0) R^{3} r^{\prime}=3 r_{g} \frac{R^{3}}{r R_{b}^{3}} r^{\prime},
$$

and then, equating this to (38), we arrive at two expressions for $F^{\prime}$ :

$$
F^{\prime}=3 r_{g} \frac{R^{3}}{r R_{b}^{3}} r^{\prime}=r_{g} \frac{3 R^{2}}{R_{b}^{3}},
$$

from which we find:

$$
r^{\prime}=\frac{r}{R}
$$

Thus, the second additional consequence of homogeneity is that this condition allows one to find $r^{\prime}$ also, which is the same for all three special velocities. 


\section{A homogeneous dust star on a hypersurface of simultaneity}

Tolman's solution inside the dust star in the comoving frame of reference (28), with taking into account the consequences of homogeneity (38) and (41), naturally coincides with earlier Friedmann's cosmological solution obtained under the same conditions. In contrast to cosmology, in astrophysics one deals with a compact object embedded in the static Schwarzschild space-time (1). Therefore, for the matching with the exterior metric, the interior solution must be expressed in the same coordinates as the static metric.

This is exactly what was done in the OS paper, where they able to find the exact solutions for the interior metric and trajectories in terms of the coordinates $r, t$, and also to sew them on the surface with the exterior static metric in the particular case when:

a) the dust ball is homogeneous in the local comoving reference frames;

b) the dust particles fall with the parabolic velocity and $f(R)=0$;

c) Tolman's solution is transformed into the Schwarzschild coordinates $r, t$.

Further, we give the details of the calculations in the OS method not only for the parabolic, but for the hyperbolic and elliptic velocities also.

\subsection{The metric and trajectories in the exterior region}

\section{a. Parabolic velocities.}

Let us consider the falling test particles outside the star $R>R_{b}$. Since the metric in the exterior region in the static reference frame is known and given by the Schwarzschild metric (1), we proceed from it and transform it from $r, t$ to $R, \tau$. We express the intervals of $r, t$ across the intervals of $R, \tau$ in the form:

$$
d t=\dot{t} d \tau+t^{\prime} d R, \quad d r=\dot{r} d \tau+r^{\prime} d R .
$$

The line element (1) then turns to $\left(\alpha \equiv 1-r_{g} / r\right)$ :

$$
\begin{aligned}
d s^{2} & =\alpha d t^{2}-\alpha^{-1} d r^{2}-r^{2} d^{2} \Omega= \\
& =\left(\alpha \dot{t}^{2}-\alpha^{-1} \dot{r}^{2}\right) d \tau^{2}-\left(\alpha^{-1} r^{\prime 2}-\alpha t^{\prime 2}\right) d R^{2}+2\left(\alpha \dot{t} t^{\prime}-\alpha^{-1} \dot{r} r^{\prime}\right) d \tau d R-r^{2} d^{2} \Omega .
\end{aligned}
$$

The expressions for the metric components in the coordinates $R, \tau$ are also known from (27) - (28) and together with (43) they give two equations for determining $\dot{t}, t^{\prime}$, as well as the metric diagonality condition:

$$
\begin{aligned}
& g_{00}(\tau, R)=\alpha \dot{t}^{2}-\alpha^{-1} \dot{r}^{2}=1, \\
& g_{01}(\tau, R)=\alpha \dot{t} t^{\prime}-\alpha^{-1} \dot{r} r^{\prime}=0 . \\
& g_{11}(\tau, R)=-\left(\alpha^{-1} r^{\prime 2}-\alpha t^{\prime 2}\right)=-r^{\prime 2},
\end{aligned}
$$

Taking into account that $F=r_{g}$ outside the star, from (4), (44) and (46) the previous expressions (7) and (10) for $\dot{r}, r^{\prime}$ and $\dot{t}$ are follow. From (46) we find the expression for $t^{\prime}$ by taking into account $r^{\prime}=R^{1 / 2} / r^{1 / 2}$ from (41):

$$
t^{\prime}=-r^{\prime} \frac{\left(r r_{g}\right)^{1 / 2}}{r-r_{g}}=-\frac{\left(r_{g} R\right)^{1 / 2}}{r-r_{g}} .
$$

This expression satisfies the diagonality condition (45), which, taking into account (10) for parabolic velocities, takes the form:

$$
\frac{t^{\prime}}{\dot{t}}=r^{\prime} \dot{r}
$$


Next, calculating from (9) and equating the result to (47), we arrive at the equation for finding $t_{0}{ }^{\prime}$ :

$$
t^{\prime}=t_{0}{ }^{\prime}+\frac{R^{3 / 2}}{r_{g}^{1 / 2}\left(R-r_{g}\right)}-\frac{r^{3 / 2} r^{\prime}}{r_{g}^{1 / 2}\left(r-r_{g}\right)}=-\frac{\left(r_{g} r\right)^{1 / 2}}{r-r_{g}} r^{\prime},
$$

which after substitution $r^{\prime}=R^{1 / 2} / r^{1 / 2}$ gives:

$$
\begin{gathered}
t_{0}{ }^{\prime}=-\frac{\left(r_{g} R\right)^{1 / 2}}{R-r_{g}}, \\
t_{0}(R)=-2 r_{g}^{1 / 2} R^{1 / 2}-r_{g} \ln \frac{R^{1 / 2}-r_{g}^{1 / 2}}{R^{1 / 2}+r_{g}^{1 / 2}} .
\end{gathered}
$$

Finally, substituting (51) into (9), we obtain the trajectory function $r(t, R)$ in the form as it was used in the OC paper:

$$
t=\frac{2}{3 r_{g}^{1 / 2}}\left(R^{3 / 2}-r^{3 / 2}\right)-2 r_{g}^{1 / 2} r^{1 / 2}+r_{g} \ln \frac{r^{1 / 2}+r_{g}^{1 / 2}}{r^{1 / 2}-r_{g}^{1 / 2}} .
$$

In the plots of the trajectories $r(t, R)$ and $r(\tau, R)$ (Fig. 1), it is clear that there is a one-to-one correspondence between the events on both curves $r(t, R)=r(\tau, R)$, and therefore both curves are asymptotic. At $t \rightarrow \infty$ both they are asymptotically approach the gravitational radius $r(t, R) \rightarrow r_{g}$ and $r[\tau(t, R)] \rightarrow r_{g}$.

The relation between the two types of time $\tau(t, R)$ follows from (6) and (52):

$$
t=\tau-2 r_{g}^{1 / 2}\left(R^{3 / 2}-3 r_{g}^{1 / 2} \tau / 2\right)^{1 / 3}+r_{g} \ln \left(\frac{\left(R^{3 / 2}-3 r_{g}^{1 / 2} \tau / 2\right)^{1 / 3}+r_{g}^{1 / 2}}{\left(R^{3 / 2}-3 r_{g}^{1 / 2} \tau / 2\right)^{1 / 3}-r_{g}^{1 / 2}}\right) .
$$

On the plot for $\tau(t, R)$ (Fig. 2) the points with $t=$ const. correspond to simultaneous events, and with $\tau=$ const. - to one-age ones. At $t \rightarrow \infty$ and $r \rightarrow r_{g}$ the moment of freezing of the proper time $\tau \rightarrow \tau_{g}$, which appear as asymptotes in Fig. 2, to which the proper times tend, we find from (6):

$$
\tau_{g}(R)=\frac{2}{3 r_{g}^{1 / 2}}\left(R^{3 / 2}-r_{g}^{3 / 2}\right) .
$$

\section{b. Hyperbolic and elliptic velocities.}

The transformation of the Schwarzschild solution (1) from $r, t$ to $R, \tau$ the hyperbolic and elliptic velocities is analogous to the parabolic case (42) - (45) with the difference only in the spatial component of the metric:

$$
g_{11}(\tau, R)=-\left(\alpha^{-1} r^{\prime 2}-\alpha t^{\prime 2}\right)=-\bar{r}^{\prime 2},
$$

where $\bar{r}^{\prime 2}=r^{\prime 2} /\left(1 \pm r_{g} / R\right)$. For $\dot{r}$ and $\dot{t}$ from (3) and (44) follow:

$$
\dot{r}=-\frac{r_{g}^{1 / 2}}{r^{1 / 2}}\left(1 \pm \frac{r}{R}\right)^{1 / 2}, \dot{t}=\frac{\left(1 \pm r_{g} / R\right)^{1 / 2}}{1-r_{g} / r}
$$

From (55) we obtain an expression for $t^{\prime}$ satisfying the diagonality condition (45): 

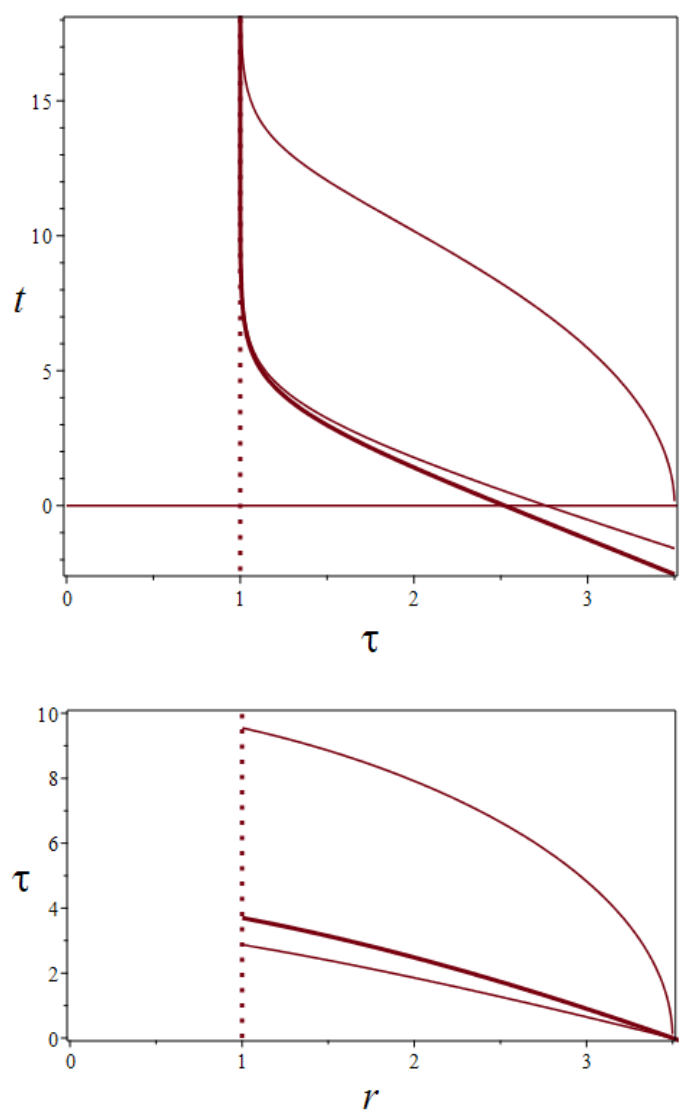

Fig.1. The worldline of a particle on the surface of the star $r=r(t)=r(\tau)$ in terms of $t$ (upper figure) and $\tau$ (lower figure) falling from $R=3.5$ at $\tau=0$ (in units $r_{g}$ ). A thick line corresponds to the parabolic, a line close to it - to the hyperbolic and an upper one to the elliptic velocities.

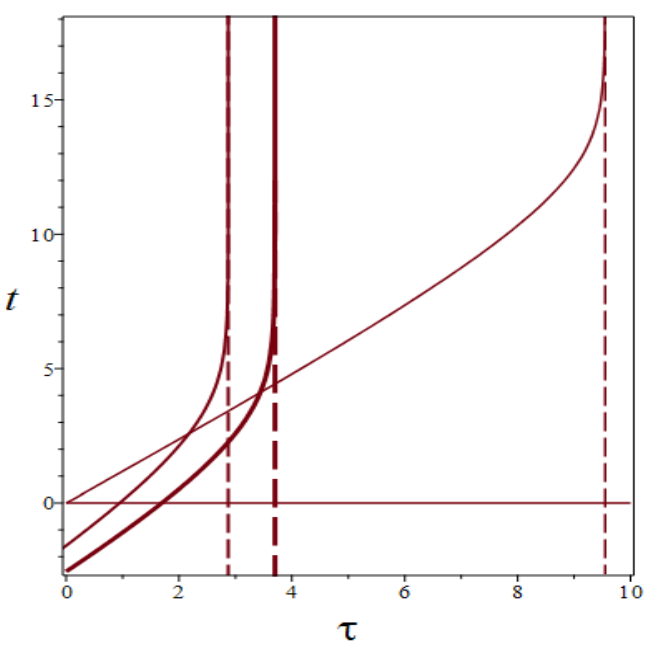

Fig 2. The relationship $\tau(t, R)$ between the proper time $\tau$ and world time $t$ for the particles from Fig.1. Dotted lines are asymptotes $\tau_{g}$ from (54), (61) - (62) to which the proper times $\tau$ tend at $t \gg r_{g}$. It can be seen that the values of $\tau$ only approach $\tau_{g}$, never reaching it at $t<\infty$, and the surface remains outside $r_{g}$ also in terms of $\tau$ too. The events on the worldline $r=r(\tau)$ in the bottom Fig. 1 are the same events as in the worldline $r=r(t)$ in the upper Fig.1, i.e. the points of two parametrizations of the same worldline correspond to each other one to one. Thus, the worldlines $r(\tau)$ on the lower Fig. 1 also are asymptotic and do not cross $r_{g}$.

$$
t^{\prime}=-r^{\prime} \frac{\left(r_{g} r\right)^{1 / 2}}{r-r_{g}}\left(\frac{R \pm r}{R+r_{g}}\right)^{1 / 2}=\frac{\dot{r} r^{\prime}}{\dot{t} \alpha^{2}} .
$$

Calculating $t^{\prime}$ from the solution (21) for the hyperbolic velocities and equating the result to (57), we arrive at the equation for $t_{0}{ }^{\prime}$ :

$$
t_{0}{ }^{\prime}=\frac{3}{2^{1 / 2}} \frac{R / r_{g}}{\left(1+R / r_{g}\right)^{1 / 2}},
$$

the solution of which has the form:

$$
t_{0}(R)=2^{1 / 2}\left(R-2 r_{g}\right)\left(1+R / r_{g}\right)^{1 / 2} .
$$

Substituting this into (21), we obtain the trajectory function in the hyperbolic case: 


$$
\begin{aligned}
& t=2 r_{g} \ln \frac{\left[r\left(R+r_{g}\right)\right]^{1 / 2}+\left[r_{g}(R+r)\right]^{1 / 2}}{\left[R\left(r-r_{g}\right)\right]^{1 / 2}}+ \\
& +\left[\left(R-2 r_{g}\right)\left(2^{1 / 2}+\ln \frac{r^{1 / 2}+(R+r)^{1 / 2}}{R^{1 / 2}}\right)-[r(r+R)]^{1 / 2}\right]\left(1+R / r_{g}\right)^{1 / 2}, f<0 .
\end{aligned}
$$

The trajectory function (22) for the elliptic velocities remains the same, since the expression $t^{\prime}$ from (57) gives $t_{0}(R) \equiv 0$ for all $R$.

The plots of the trajectories $r(t, R)$ and $r(\tau, R)$ are also shown in Fig. 1. It can be seen from the Fig. 1 that in each case both curves are asymptotic, i.e. at $t \rightarrow \infty$ both they are only approaching the gravitational radius $r(t, R) \equiv r(\tau, R) \rightarrow r_{g}$.

At $t \rightarrow \infty$ and $r \rightarrow r_{g}$ the freezing moments of proper times $\tau \rightarrow \tau_{g}$ at the hyperbolic and elliptic velocities we find from (15) and (16):

$$
\begin{aligned}
\tau_{g} & =\frac{R^{1 / 2}}{r_{g}^{1 / 2}}\left[2^{1 / 2} R-\left[r_{g}\left(R+r_{g}\right)\right]^{1 / 2}\right]+\frac{R^{3 / 2}}{r_{g}^{1 / 2}} \ln \left(\frac{r_{g}^{1 / 2}+\left(R+r_{g}\right)^{1 / 2}}{R^{1 / 2}\left(1+2^{1 / 2}\right)}\right), \quad f>0 \\
\tau_{g} & =\frac{R^{1 / 2}}{r_{g}^{1 / 2}}\left[r_{g}\left(R-r_{g}\right)\right]^{1 / 2}+\frac{R^{3 / 2}}{r_{g}^{1 / 2}} \arccos \left(r_{g}^{1 / 2} / R^{1 / 2}\right), \quad f<0 .
\end{aligned}
$$

From the plot of the relation of two times $\tau(t, R)$ in Fig. 2 we see that at $t \rightarrow \infty$ the proper times of the layers $R$ tend to their asymptotes $\tau_{g}(R)$ from (61) - (62).

In the literature it is often used a parametric representation for variables and trajectory equations, and in Appendix 1 the main results are also presented in the such form.

\subsection{The metric and trajectories in the interior region}

In the previous Section, the solutions in terms of $r, t$ coordinates were obtained in terms of $R, \tau$. Here, following OS, we will perform the inverse transformation - from the Tolman's interior solution (28) in terms of $R, \tau$ we will obtain the solution in terms of the Schwarzschild coordinates $r, t$. This is necessary both for matching with the exterior metric (1) and for determining the instantaneous structure of the star as a whole on the hypersurface of simultaneity $t=$ const .

The line element inside the star can be in general with a non-diagonal metric. However, firstly, this metric must be diagonal on the surface to be matched with the exterior metric. Secondly, the instantaneous structure of the star as an extended object must be given on a single hypersurface of simultaneity coinciding on the surface with the hypersurface of world time. This also suggests the existence of clocks in the entire volume of the star, which are synchronized and show the same world time as on the surface.

All this is realized in a natural way only for the diagonal metric and it is very difficult, if at all possible, for a non-diagonal metric. Therefore, we need to test the possibility of the existence of an exact solution with the diagonal metric. If such a solution can be found, then all other solutions with the non-diagonal metric will turn out to be just the same simple solution, but transformed into more inconvenient or inadequate coordinates.

Thus, we write down a line element with diagonal metric:

$$
d s^{2}=e^{\nu(r, t)} d t^{2}-e^{\lambda(r, t)} d r^{2}-r^{2} d^{2} \Omega, \quad r \leq r_{b},
$$

the matching on the surface of which should give $e^{v\left(r_{b}, t\right)}=e^{-\lambda\left(r_{b}, t\right)}=1-r_{g} / r_{b}$. From (42) it follows: 


$$
\begin{aligned}
& d s^{2}=e^{v} d t^{2}-e^{\lambda} d r^{2}-r^{2} d^{2} \Omega= \\
& =\left(e^{v} \dot{t}^{2}-e^{\lambda} \dot{r}^{2}\right) d \tau^{2}-\left(e^{\lambda} r^{\prime 2}-e^{v} t^{\prime 2}\right) d R^{2}+2\left(e^{v} \dot{t} t^{\prime}-e^{\lambda} \dot{r} r^{\prime}\right) d \tau d R-r^{2} d^{2} \Omega .
\end{aligned}
$$

Notice that the OS method differs from other attempts to obtain solutions with diagonalization of the interior metric $g_{01}(r, t)=0$ in that the diagonality of the required metric is considered as the additional requirement and further exact solutions are found that satisfy this condition. The coordinate time $t$ is the same for all layers and coincides on the surface with the exterior world time.

In accordance with (27) we obtain the conditions:

$$
\begin{aligned}
& g_{00}(\tau, R)=e^{v} \dot{t}^{2}-e^{\lambda} \dot{r}^{2}=1, \\
& g_{11}(\tau, R)=-\left(e^{\lambda} r^{\prime 2}-e^{v} t^{\prime 2}\right)=-\bar{r}^{\prime 2}, \\
& g_{01}(\tau, R)=e^{v} \dot{t} t^{\prime}-e^{\lambda} \dot{r} r^{\prime}=0 .
\end{aligned}
$$

where $\bar{r}^{\prime 2}=r^{\prime 2} /(1+f)$. The first two of them give for two components of the metric:

$$
e^{\lambda}=\frac{t^{\prime 2}+\dot{t}^{2} \bar{r}^{\prime 2}}{\dot{t}^{2} r^{\prime 2}-t^{\prime 2} \dot{r}^{2}}, \quad e^{v}=\frac{r^{\prime 2}+\bar{r}^{\prime 2} \dot{r}^{2}}{\dot{t}^{2} r^{\prime 2}-t^{\prime 2} \dot{r}^{2}},
$$

and the third one (67) expresses the diagonality of the initial metric, which, taking into account (68), has the form:

$$
g_{01}=\frac{\dot{t} t^{\prime}\left(r^{\prime 2}+\bar{r}^{\prime 2} \dot{r}^{2}\right)-\left(t^{\prime 2}+\dot{t}^{2} \bar{r}^{\prime 2}\right) \dot{r} r^{\prime}}{\dot{t}^{2} r^{\prime 2}-t^{\prime 2} \dot{r}^{2}}=0
$$

Writing this condition as an equation for $t^{\prime}$ :

$$
t^{\prime 2} \dot{r}-t^{\prime} \dot{t} r^{\prime}\left(1+\frac{\dot{r}^{2}}{1+f}\right)+\dot{t}^{2} \dot{r} \bar{r}^{\prime 2}=0,
$$

we obtain two solutions:

$$
t^{\prime}=\frac{\dot{t} r^{\prime}}{2 \dot{r}}\left(\left[1+\dot{r}^{2} /(1+f)\right] \pm\left[1-\dot{r}^{2} /(1+f)\right]\right) .
$$

The first one of these (with a plus) $t^{\prime}=\dot{t}^{\prime} / \dot{r}$ is unphysical, since it diverges at $\dot{r} \rightarrow 0$, and the second one (with a minus sign) gives the diagonality condition of the interior metric:

$$
\frac{t^{\prime}}{\dot{t}}=\frac{\dot{r} r^{\prime}}{1+f} \text {. }
$$

Excluding $t^{\prime}$ by means of (72), we simplify the expression for the metric (68):

$$
e^{\lambda}=\frac{1}{1-F / r}, \quad e^{v}=\frac{1+f}{\dot{t}^{2}(1-F / r)} .
$$

a. Parabolic velocities.

Setting $f=0$ in (72) we obtain the diagonality condition for the parabolic velocities:

$$
t^{\prime} / \dot{t}=r^{\prime} \dot{r}
$$

The OS solution for the metric [4], following from (73) and (74), has the form:

$$
e^{\lambda}=\frac{1}{1-\dot{r}^{2}}, \quad e^{v}=\frac{1}{\dot{t}^{2}\left(1-\dot{r}^{2}\right)} .
$$


Thus, $r^{\prime}$ is known from (41), the expressions for $\tau(r, R), r(\tau, R)$ and $\dot{r}$ we obtain from (31) and (38):

$$
\tau=\frac{2}{3 r_{g, R}^{1 / 2}}\left(R^{3 / 2}-r^{3 / 2}\right), \quad r(\tau, R)=R\left(1-\frac{3 r_{g}^{1 / 2}}{2 R_{b}^{3 / 2}} \tau\right)^{2 / 3}, \quad \dot{r}=-\frac{r_{g, R}^{1 / 2}}{r^{1 / 2}} .
$$

To determine the trajectory equation $r(t, R)$ in the OC method (Oppenheimer \& Snyder, 1939), it is necessary to introduce a new variable $y$ :

$$
t=M(y),
$$

where the form of the function $M$ is determined from the matching condition with the exterior metric. The diagonality condition (74) then takes the form:

$$
\frac{t^{\prime}}{\dot{t}}=\frac{y^{\prime}}{\dot{y}}=\dot{r} r^{\prime}=-\frac{\left(r_{g, R} r\right)^{1 / 2}}{R} \text {. }
$$

The solution of this equation for $y$, found by the OS, has the form:

$$
y(R, \tau)=\frac{1}{2}\left(\frac{R^{2}}{R_{b}^{2}}-1\right)+\frac{R_{b}}{R r_{g}} r .
$$

The derivation of this solution is presented in Appendix 2.

On the surface $y_{b}=y\left(R_{b}\right)=r / r_{g}$ and here the function $M\left(y_{b}\right)$ must coincide with the right-hand side of (52). Therefore, by substituting $R \rightarrow R_{b}$ and $r \rightarrow r_{g} y$ in (52), OS have obtained the trajectory function for the particles inside the star:

$$
t=\frac{2}{3 r_{g}^{1 / 2}}\left(R_{b}^{3 / 2}-r_{g}^{3 / 2} y^{3 / 2}\right)-2 r_{g} y^{1 / 2}+r_{g} \ln \frac{y^{1 / 2}+1}{y^{1 / 2}-1} .
$$

For $\dot{t}$ и $t^{\prime}$ this equation, by taking into account $y^{\prime}=R / R_{b}^{2}$ и $\dot{y}=-\left(R / r r_{g} R_{b}\right)^{1 / 2}$, gives:

$$
\dot{t}=-\frac{r_{g} y^{3 / 2} \dot{y}}{y-1}=\left(\frac{r_{g} R}{R_{b} r}\right)^{1 / 2} \frac{y^{3 / 2}}{y-1}, \quad t^{\prime}=-\frac{r_{g} y^{3 / 2} y^{\prime}}{y-1}=-\frac{r_{g} R}{R_{b}^{2}} \frac{y^{3 / 2}}{y-1} .
$$

and the diagonality condition (78) is satisfied.

b. Hyperbolic and elliptic velocities.

In this case, from (66), (32) and (33) we have:

$$
f= \pm \frac{F}{R}= \pm \frac{r_{g, R}}{R}, \quad \dot{r}=-\frac{r_{g, R}^{1 / 2}}{r^{1 / 2}}(1 \pm r / R)^{1 / 2} .
$$

Substituting this into (73) and (82), we obtain:

$$
e^{\lambda}=\frac{1}{1-r_{g, R} / r}, \quad e^{v}=\dot{t}^{-2} \frac{1 \pm r_{g, R} / R}{1-r_{g, R} / r} .
$$

By introducing the variable $y$ as in (77), we write the diagonal condition (72) in the form:

$$
\frac{t^{\prime}}{\dot{t}}=\frac{y^{\prime}}{\dot{y}}=\frac{\dot{r} r^{\prime}}{1 \pm r_{g, R} / R}=-\frac{\left(r_{g, R} r\right)^{1 / 2}}{R \pm r_{g, R}}(1 \pm r / R)^{1 / 2}
$$

The solutions of this equation with respect to $y$ have the form:

$$
y(R, r)= \pm \frac{R_{b}}{r_{g}} \ln \left(\frac{\left(1 \pm r_{g, R} / R\right)^{1 / 2}(1 \pm r / R)}{\left(1 \pm r_{g} / R_{b}\right)^{1 / 2}}\right),
$$




$$
r= \pm R\left(\frac{\left(1 \pm r_{g} / R_{b}\right)^{1 / 2}}{\left(1 \pm r_{g, R} / R\right)^{1 / 2}} e^{ \pm y r_{g} / R_{b}}-1\right) .
$$

The derivation of these solutions is also presented in Appendix 2. At $R=R_{b}$ :

$$
y\left(R_{b}, r\right) \equiv y_{b}= \pm\left(R_{b} / r_{g}\right) \ln \left(1 \pm r / R_{b}\right) .
$$

On the surface, the function $M\left(y_{b}\right)$ must coincide with the right-hand side of (22). Making in (22) the substitutions $R \rightarrow R_{b}$ and $r=r_{y}$, where $r_{y}$ is determined from the correspondence with (87) as:

$$
r_{ \pm}= \pm R_{b}\left[\exp \left( \pm y r_{g} / R_{b}\right)-1\right]
$$

we obtain the trajectory function $r(t, R)$ in the implicit form $t(R, r)\left(b=R_{b} / r_{g}\right)$ :

$$
\begin{aligned}
& t=2 r_{g} \ln \frac{\left[r_{+}\left(R_{b}+r_{g}\right)\right]^{1 / 2}+\left[r_{g}\left(R_{b}+r_{+}\right)\right]^{1 / 2}}{\left[R_{b}\left(r_{+}-r_{g}\right)\right]^{1 / 2}}+ \\
& +\left[\left(R_{b}-2 r_{g}\right)\left(2^{1 / 2}+\ln \frac{r_{+}^{1 / 2}+\left(R_{b}+r_{+}\right)^{1 / 2}}{R_{b}^{1 / 2}}\right)-\left[r_{+}\left(r_{+}+R_{b}\right)\right]^{1 / 2}\right]\left(\frac{R_{b}}{r_{g}}+1\right)^{1 / 2}, f>0, \\
& t=2 r_{g} \ln \frac{\left[r_{-}\left(R_{b}-r_{g}\right)\right]^{1 / 2}+\left[r_{g}\left(R_{b}-r_{-}\right)\right]^{1 / 2}}{\left[R_{b}\left(r_{-}-r_{g}\right)\right]^{1 / 2}}+ \\
& +\left(\left(R_{b}+2 r_{g}\right) \arccos \left(r_{-}^{1 / 2} / R_{b}^{1 / 2}\right)+\left[r_{-}\left(R_{b}-r_{-}\right)\right]^{1 / 2}\right)\left(\frac{R_{b}}{r_{g}}-1\right)^{1 / 2}, f<0
\end{aligned}
$$

The derivative $\dot{t}$ have the form:

$$
\dot{t}= \pm \frac{r_{ \pm}^{1 / 2}\left(R_{b} \pm r_{g}\right)^{1 / 2}}{r_{g}^{1 / 2}\left(R_{b} \pm r_{ \pm}\right)^{1 / 2}} \frac{\dot{r}_{ \pm}}{1 \pm r_{g} / r_{ \pm}}, \quad \dot{r}_{ \pm}=\dot{y} \frac{R r_{g}}{R_{b}} \frac{\left(1 \pm r_{g} / R_{b}\right)^{1 / 2}}{\left(1 \pm r_{g, R} / R\right)^{1 / 2}} e^{ \pm y r_{g} / R_{b}}
$$

\section{The internal structure and evolution of a dust star}

\subsection{Trajectories of particles in layers and their asymptotes}

\section{a. Parabolic velocities.}

The plots of the trajectories of the dust particles inside the star $r(t, R)$ for the parabolic solution of OS (79) - (80) are shown in Fig. 3-4. In Fig. 4 shows the asymptotes $r(\infty, R)$ to which the trajectories of the particles of the layers tend at $t \rightarrow \infty$. They are parallel to the worldline of the center and are placed almost equidistantly, condensing only near the surface. The closure is insignificant and concerns only those layers that were initially close to the surface. An expression for the asymptotes of worldlines inside the star can be found in the following way.

At $t \rightarrow \infty$ the surface of the star asymptotically approaches the gravitational radius $r_{b} \rightarrow r_{g}$ and in (9) it dominates the growing logarithmic term, which gives on the surface:

$$
\begin{gathered}
t \simeq-r_{g} \ln \left(\frac{r_{b}^{1 / 2}-r_{g}^{1 / 2}}{r_{b}^{1 / 2}+r_{g}^{1 / 2}}\right), \quad e^{-t / r_{g}} \simeq \frac{r_{b}-r_{g}}{\left(r_{b}^{1 / 2}+r_{g}^{1 / 2}\right)^{2}} \simeq \frac{1}{4}\left(\frac{r_{b}}{r_{g}}-1\right), \\
r_{b}(t) \simeq r_{g}\left(1+4 e^{-t / r_{g}}\right)>r_{g} .
\end{gathered}
$$



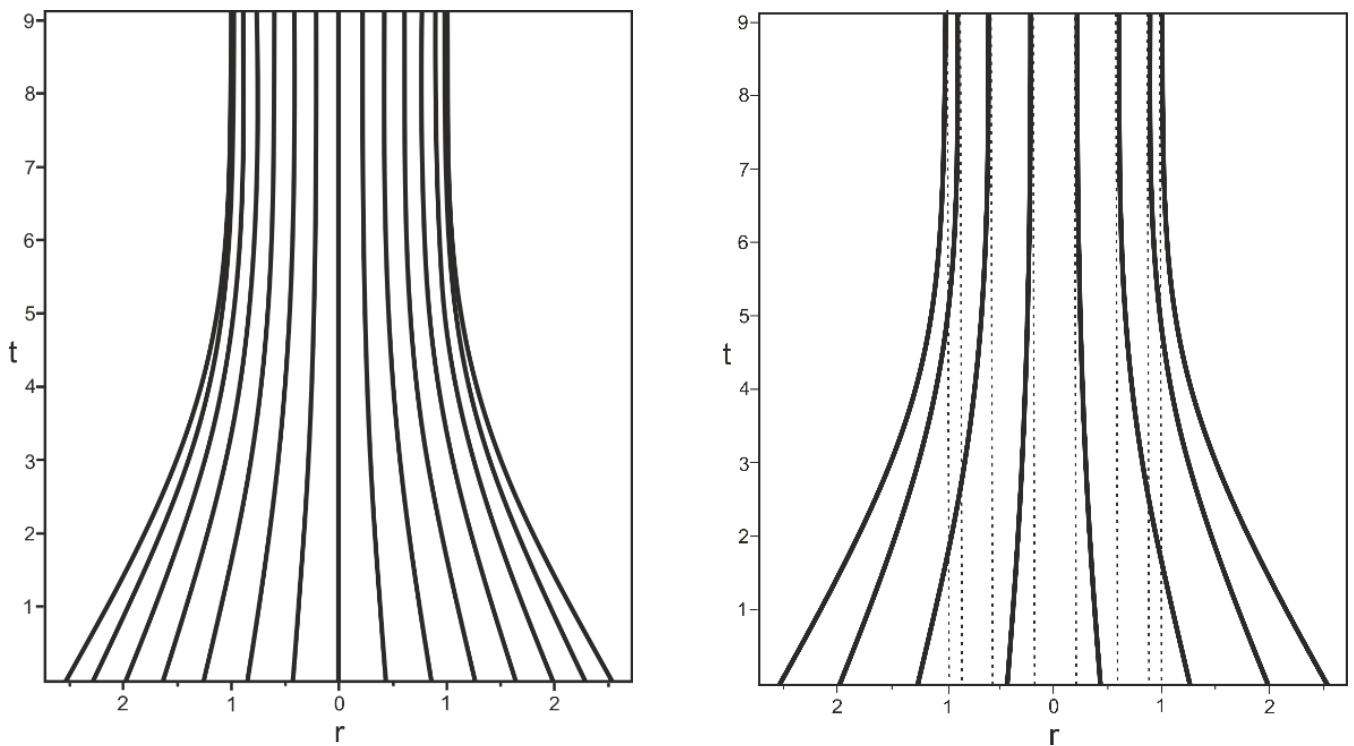

Fig.3. The OS worldlines of the dust star's particles Fig. 4. The worldlines of particles in the layers along the star's diameter at the parabolic velocity of the dust ball from Fig.3. At $t \gg r_{g}$ a surface and $R_{b}=3.5$ at $\tau=0$ (in units $r_{g}$ ) according Eqs.

(79)-(80) At large $t$ the worldlines remain timelike, the surface freezes outside the gravitational radius, the internal layers freeze near own asymptotes given asymptotically approaches $r_{g}$, but not cross it. Inner layers freeze near their asymptotes from Eq. (95) shown by dotter verticals. by Eq. (95)

In the internal layers, the logarithmic contribution to (80) also grows, but now at $y \rightarrow 1$ and this gives for the asymptotes of the layers $r(t, R) \rightarrow r_{\infty}(R)$ the equation:

$$
\left.y(t, R)\right|_{t \rightarrow \infty}=y_{\infty}(R)=\frac{1}{2}\left(\frac{R^{2}}{R_{b}^{2}}-1\right)+\frac{R_{b}}{r_{g} R} r_{\infty}(R)=1,
$$

from which we find the required result:

$$
r_{\infty}(R)=R \frac{3 r_{g}}{2 R_{b}}\left(1-\frac{R^{2}}{3 R_{b}^{2}}\right) .
$$

As we see, the lines of asymptotes of the internal layers of the star are located almost equidistant (Fig. 4) and only the layers near the surface are densified due to the factor $-r_{g} R^{3} / 2 R_{b}^{3}$.

The proper time on each layer freezes at $\tau_{\infty}(R)$, the value of which we find by substituting $r_{\infty}(R)$ into (76):

$$
\tau_{\infty}(R)=\frac{2 R_{b}^{3 / 2}}{3 r_{g}^{1 / 2}}-\frac{3^{1 / 2} r_{g}}{2^{1 / 2}}\left(1-\frac{R^{2}}{3 R_{b}^{2}}\right)^{3 / 2} .
$$

The center freezes before all layers the moment $\tau_{\infty}(0)$ and the surface freezes later than all layers at $\tau_{\infty}\left(R_{b}\right)$ :

$$
\tau_{\infty}(0)=\frac{2}{3}\left(\frac{R_{b}^{3 / 2}}{r_{g}^{3 / 2}}-\frac{3^{3 / 2}}{2^{3 / 2}}\right) r_{g}, \quad \tau_{\infty}\left(R_{b}\right)=\frac{2}{3}\left(\frac{R_{b}^{3 / 2}}{r_{g}^{3 / 2}}-1\right) r_{g} .
$$


The interval of proper time, during which all the layers of the dust star become frozen, is therefore equal to the difference between the moments of freezing of the surface and the center:

$$
\tau_{\infty}\left(R_{b}\right)-\tau_{\infty}(0)=\frac{2}{3}\left(\frac{3^{3 / 2}}{2^{3 / 2}}-1\right) r_{g} \approx 0.558 r_{g} .
$$

For a star with $10 M_{\odot}$ and $r_{g} \simeq 30 \mathrm{~km}$ the difference between the moments showing by frozen local clocks on the surface and at the center will be about 0.1 milliseconds. In the world time this interval, of course, is sufficiently larger.

Let us consider the dependence on $t$ of the basic variables at large $t$. From the asymptotes $t$ :

$$
t \simeq-r_{g} \ln \left(\frac{y-1}{\left(y^{1 / 2}+1\right)^{2}}\right) \simeq-r_{g} \ln \left(\frac{y-1}{4}\right)
$$

we obtain:

$$
\begin{aligned}
& y=\frac{1}{2}\left(\frac{R^{2}}{R_{b}^{2}}-1\right)+\frac{R_{b}}{R r_{g}} r \simeq 1+4 e^{-t / r_{g}}, \\
& r \simeq R \frac{3 r_{g}}{2 R_{b}}\left(1-\frac{R^{2}}{3 R_{b}^{2}}+\frac{8}{3} e^{-t / r_{g}}\right) .
\end{aligned}
$$

Substituting this into (76), we then find the dependence of the moments of proper time inside the star $\tau(t, R)$ from $t$ :

$$
\tau=\frac{2 R_{b}^{3 / 2}}{3 r_{g}^{1 / 2}}-\frac{3^{1 / 2} r_{g}}{2^{1 / 2}}\left(1-\frac{R^{2}}{3 R_{b}^{2}}+\frac{8}{3} e^{-t / r_{g}}\right)^{3 / 2} .
$$

At $t \rightarrow \infty$ this expression goes into (96).

\section{b. Hyperbolic and elliptic velocities.}

The trajectory plots $r(t, R)$ in accordance with the solutions for the hyperbolic and elliptic velocities (85) and (89) - (90) are shown in Fig. 5-6. It can be seen from the figures that at $t \rightarrow \infty$ the trajectories of the particles in different layers $R$, as in the parabolic case, also tend to their own asymptotes $r(\infty, R)$. Let us find these asymptotes.

At $t \rightarrow \infty$ in these two cases also $r_{b} \rightarrow r_{g}$ and in (89) - (90) the increasing logarithmic terms dominate, which gives:

$$
\begin{aligned}
& t \simeq-r_{g} \ln \left( \pm \frac{R_{b}}{r_{g}}\left[\exp \left( \pm y r_{g} / R_{b}\right)-1\right]-1\right)=-r_{g} \ln \left(\frac{r_{ \pm}}{r_{g}}-1\right), \\
& r_{ \pm}= \pm R_{b}\left[\exp \left( \pm y r_{g} / R_{b}\right)-1\right] .
\end{aligned}
$$

The asymptotes $y \rightarrow y_{\infty}$ at $t \rightarrow \infty$ follows from (103):

$$
\begin{gathered}
\pm \frac{R_{b}}{r_{g}}\left[\exp \left( \pm y_{\infty} r_{g} / R_{b}\right)-1\right]=1, \\
y_{\infty}= \pm \frac{R_{b}}{r_{g}} \ln \left(1 \pm \frac{r_{g}}{R_{b}}\right) .
\end{gathered}
$$



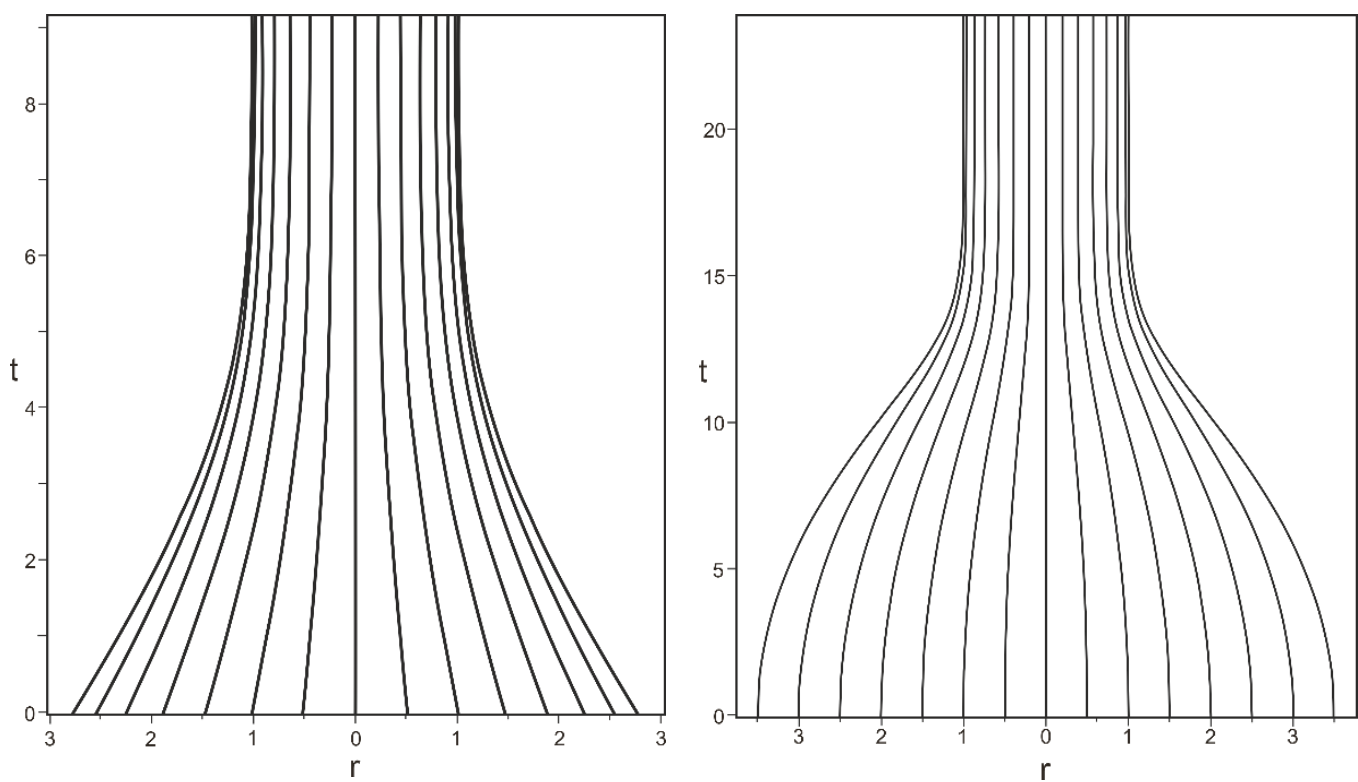

Fig. 5. The OS worldlines of the dust star's particles Fig. 6. The OS worldlines of the dust star's along the star's diameter at the hyperbolic velocity particles along the star's diameter at the elliptic and $R_{b}=3.5$ at $\tau=0$ (in units $r_{g}$ ) according Eqs. velocity and $R_{b}=3.5$ at $\tau=0$ (in units $r_{g}$ ) (85), (88) and (89). At large $t$ the worldlines remain according Eqs. (85), (88) and (90). At large $t$ the timelike, the surface freezes outside the gravitational worldlines remain timelike, the surface freezes radius, the internal layers freeze near own outside the gravitational radius, the internal asymptotes given by Eq. (107). layers freeze near own asymptotes given by Eq. (107).

Substituting this into (86), we find the asymptotes for the internal layers $r_{\infty}(R)$ :

$$
r_{\infty}(R)= \pm R\left(\frac{\left(1 \pm r_{g} / R_{b}\right)^{3 / 2}}{\left(1 \pm r_{g} R^{2} / R_{b}^{3}\right)^{1 / 2}}-1\right) .
$$

At $R_{b} \gg r_{g}$ this formula is transformed into (95). As we see, the lines of the asymptotes of the layers at the hyperbolic and elliptic velocities are also parallel to the worldline of the center, are spaced from each other almost equidistantly, condensing near the surface only.

The moments of freezing of proper time on each layer $\tau_{\infty}(R)$ we find by substituting $r_{\infty}(R)$ in (34) - (35). For hyperbolic velocities this gives:

$$
\begin{aligned}
& \tau_{g}(R)=\frac{R_{b}^{3 / 2}}{r_{g}^{1 / 2}}\left[2^{1 / 2}-\left[\frac{\left(1+r_{g} / R_{b}\right)^{3 / 2}}{\left(1+r_{g} R^{2} / R_{b}^{3}\right)^{1 / 2}}\left(\frac{\left(1+r_{g} / R_{b}\right)^{3 / 2}}{\left(1+r_{g} R^{2} / R_{b}^{3}\right)^{1 / 2}}-1\right)\right]^{1 / 2}\right]+ \\
& +\frac{R_{b}^{3 / 2}}{r_{g}^{1 / 2}} \ln \left(\frac{1}{1+2^{1 / 2}}\left[\left(\frac{\left(1+r_{g} / R_{b}\right)^{3 / 2}}{\left(1+r_{g} R^{2} / R_{b}^{3}\right)^{1 / 2}}-1\right)^{1 / 2}+\frac{\left(1+r_{g} / R_{b}\right)^{3 / 4}}{\left(1+r_{g} R^{2} / R_{b}^{3}\right)^{1 / 4}}\right]\right) .
\end{aligned}
$$

The moments of freezing of the center $\tau_{\infty}(0)$ and the surface $\tau_{\infty}\left(R_{b}\right)>\tau_{\infty}(0)$ are equal to: 


$$
\begin{aligned}
& \tau_{\infty}(0)=\frac{R_{b}^{3 / 2}}{r_{g}^{1 / 2}}\left[2^{1 / 2}-\left[\left(1+r_{g} / R_{b}\right)^{3 / 2}\left(\left(1+r_{g} / R_{b}\right)^{3 / 2}-1\right)\right]^{1 / 2}\right]+ \\
& +\frac{R_{b}^{3 / 2}}{r_{g}^{1 / 2}} \ln \left(\frac{1}{1+2^{1 / 2}}\left[\left(\left(1+r_{g} / R_{b}\right)^{3 / 2}-1\right)^{1 / 2}+\left(1+r_{g} / R_{b}\right)^{3 / 4}\right]\right), \\
& \tau_{g}\left(R_{b}\right)=\frac{R_{b}^{3 / 2}}{r_{g}^{1 / 2}}\left[2^{1 / 2}-\left[\left(1+r_{g} / R_{b}\right) r_{g} / R_{b}\right]^{1 / 2}\right]+ \\
& +\frac{R_{b}^{3 / 2}}{r_{g}^{1 / 2}} \ln \left(\frac{1}{1+2^{1 / 2}}\left[\left(r_{g} / R_{b}\right)^{1 / 2}+\left(1+r_{g} / R_{b}\right)^{1 / 2}\right]\right) .
\end{aligned}
$$

For elliptic velocities, the freezing moments are given by the expression:

$$
\begin{aligned}
& \tau_{\infty}(R)=\frac{R_{b}^{3 / 2}}{r_{g}^{1 / 2}}\left[\left(1-\frac{B}{A_{R}}\right)^{1 / 2} \frac{B^{1 / 2}}{A_{R}^{1 / 2}}+\arccos \frac{r_{g}^{1 / 2}}{R_{b}^{1 / 2}}\right], \\
& A_{R}=\left(1-r_{g} R^{2} / R_{b}^{3}\right)^{1 / 2}, \quad B=\left(1-r_{g} / R_{b}\right)^{3 / 2} .
\end{aligned}
$$

The moments of freezing of the center $\tau_{\infty}(0)$ and the surface $\tau_{\infty}\left(R_{b}\right)>\tau_{\infty}(0)$ are equal in this case to:

$$
\begin{aligned}
& \tau_{\infty}(0)=\frac{R_{b}^{3 / 2}}{r_{g}^{1 / 2}}\left([B(1-B)]^{1 / 2}+\arcsin \left(B^{1 / 2}\right)\right), \\
& \tau_{\infty}\left(R_{b}\right)=\frac{R_{b}^{3 / 2}}{r_{g}^{1 / 2}}\left[\left(1-\frac{r_{g}}{R_{b}}\right)^{1 / 2} \frac{r_{g}^{1 / 2}}{R_{b}^{1 / 2}}+\arccos \frac{r_{g}^{1 / 2}}{R_{b}^{1 / 2}}\right] .
\end{aligned}
$$

Using the asymptotes of $t$ :

$$
t \simeq-r_{g} \ln \left( \pm \frac{R_{b}}{4 r_{g}}\left[\exp \left( \pm y r_{g} / R_{b}\right)-1\right]-1 / 4\right),
$$

for the dependence on $t$ of the basic variables at large $t$ we obtain:

$$
\begin{gathered}
y \simeq \pm \frac{R_{b}}{r_{g}} \ln \left[1 \pm \frac{r_{g}}{R_{b}}\left(1+4 e^{-t / r_{g}}\right)\right], \\
r_{ \pm}= \pm R\left(\frac{\left(1 \pm r_{g} / R_{b}\right)^{1 / 2}}{\left(1 \pm r_{g, R} / R\right)^{1 / 2}}\left(1 \pm \frac{r_{g}}{R_{b}}\left(1+4 e^{-t / r_{g}}\right)\right)-1\right) .
\end{gathered}
$$

In the limit $t \rightarrow \infty$ the formulas (115) - (116) turn to (106)-(107).

\subsection{The interior metric and its asymptotes}

a. Parabolic velocities.

In the OS exact solution, the components of the metric (75), taking into account (79) and (81), become:

$$
\begin{aligned}
e^{\lambda(t, R)} & =\left(1-r_{g, R} / r\right)^{-1}, \\
e^{\nu(t, R)} & =\frac{\left[1+\left(r_{g, R} / 2 r\right)\left(1-3 R_{b}^{2} / R^{2}\right)\right]^{2}}{\left[1+\left(r_{g, R} / 2 r\right)\left(1-R_{b}^{2} / R^{2}\right)\right]^{3}} \frac{1}{1-r_{g, R} / r} .
\end{aligned}
$$

The line element, respectively, has the form: 


$$
d s^{2}=\frac{\left[1+\left(r_{g, R} / 2 r\right)\left(1-3 R_{b}^{2} / R^{2}\right)\right]^{2}}{\left[1+\left(r_{g, R} / 2 r\right)\left(1-R_{b}^{2} / R^{2}\right)\right]^{3}} \frac{d t^{2}}{1-r_{g, R} / r}-\frac{d r^{2}}{1-r_{g, R} / r}-r^{2} d^{2} \Omega .
$$

On the surface $R=R_{b}$, this line element turns to the Schwarzschild line element (1). In the center of the star $R=r=0$ the spatial metric is trivial $e^{\lambda(0, t)}=1$.

At $t \gg r_{g}$ the metric (117), taking into account (101), has the form:

$$
e^{\lambda(t, R)} \simeq\left[1-\frac{R^{2}}{R_{b}^{2}}\left(4 e^{-t / r_{g}}+\frac{1}{2}\left(3-\frac{R^{2}}{R_{b}^{2}}\right)\right)^{-1}\right]^{-1},
$$

or in a more compact form:

$$
e^{\lambda(t, R)} \simeq \frac{8 e^{-t / r_{g}}+3-R^{2} / R_{b}^{2}}{8 e^{-t / r_{g}}+3-3 R^{2} / R_{b}^{2}} .
$$

At the center and on the surface its value is equal to:

$$
e^{\lambda(t, 0)}=1, \quad e^{\lambda\left(t, R_{b}\right)} \simeq e^{t / r_{g}} / 4 .
$$

At $t \rightarrow \infty$ the metric from (121) tends to:

$$
e^{\lambda(\infty, R)}=\frac{1-R^{2} / 3 R_{b}^{2}}{1-R^{2} / R_{b}^{2}} .
$$

At $t \gg r_{g}$ the time component of the metric (75), taking into account (81) and (101), has the form:

$$
e^{\nu(t, R)} \simeq 16 e^{\lambda} e^{-2 t / r_{g}}\left(4 e^{-t / r_{g}}+\frac{1}{2}\left(3-\frac{R^{2}}{R_{b}^{2}}\right)\right)
$$

or in the expanded form:

$$
e^{v(t, R)} \simeq e^{-2 t / r_{g}} \frac{\left[8 e^{-t / r_{g}}+\left(3-R^{2} / R_{b}^{2}\right)\right]^{2}}{e^{-t / r_{g}}+3\left(1-R^{2} / R_{b}^{2}\right) / 8} .
$$

At the center and on the surface its value is equal to:

$$
e^{v(t, 0)} \simeq 24 e^{-2 t / r_{g}}, \quad e^{v\left(t, R_{b}\right)} \simeq 4 e^{-t / r_{g}} .
$$

At $t \rightarrow \infty$ the metric from (125) tends to:

$$
e^{v(\infty, R)}=24 e^{-2 t / r_{g}} \frac{\left(1-R^{2} / 3 R_{b}^{2}\right)^{2}}{1-R^{2} / R_{b}^{2}} .
$$

\section{b. Hyperbolic and elliptic velocities.}

In these cases, the components of the metric (83), taking into account (91) and (115), have the form:

$$
e^{\lambda(t, R)}=\left(1-r_{g, R} / r\right)^{-1}, \quad e^{\nu(t, R)}=\frac{e^{\lambda}}{\dot{t}^{2}}\left(1 \pm r_{g, R} / R\right) .
$$

On the surface $R=R_{b}$ this metric turns to the Schwarzschild metric (1). At the center of the star $R=r=0$ the spatial metric is trivial $e^{\lambda(0, t)}=1$.

At large $t$, the metric (128), taking into account (116), has the form: 


$$
e^{-\lambda(R, t)}=1 \mp \frac{r_{g, R}}{R}\left[\frac{\left(1 \pm r_{g} / R_{b}\right)^{1 / 2}}{\left(1 \pm r_{g, R} / R\right)^{1 / 2}}\left(1 \pm \frac{r_{g}}{R_{b}}\left(1+4 e^{-t / r_{g}}\right)\right)-1\right]^{-1}
$$

At the center and on the surface its value is equal to:

$$
e^{\lambda(t, 0)}=1, \quad e^{\lambda\left(t, R_{b}\right)} \simeq e^{t / r_{g}} / 4 .
$$

At $t \rightarrow \infty$ the metric tends to its asymptote:

$$
e^{-\lambda(R, \infty)}=1 \mp \frac{r_{g, R}}{R}\left[\frac{\left(1 \pm r_{g} / R_{b}\right)^{1 / 2}}{\left(1 \pm r_{g, R} / R\right)^{1 / 2}}\left(1 \pm \frac{r_{g}}{R_{b}}\right)-1\right]^{-1} .
$$

At large $t$ the value of the time component of the metric (128) at the center and on the surface, by taking into account (91) and (130), becomes equal to:

$$
e^{v(t, 0)} \sim e^{-2 t / r_{g}}, \quad e^{v\left(t, R_{b}\right)} \simeq 4 e^{-t / r_{g}} .
$$

\section{Conclusion}

In the paper, the collapse of a homogeneous dust star and its structure at the given moment of the world time in the framework of the OS method are studied in all three special velocities - parabolic, hyperbolic and elliptic. The plots of the trajectories of the star's particles (Figs. 5-8) clearly show the internal structure of the dust star on the hypersurfaces of simultaneity. At large $t$, the worldlines of particles in all layers approach their asymptotes, which are placed almost equidistantly from the center and are condensed only near the surface. The surface asymptotically approaches the gravitational radius, always remaining outside of it.

This shows that the frozen star picture refers not only to the surface, but also to the structure of the entire dust star. Due to the uniqueness and invariance of worldlines in general relativity, this picture also is unique and invariant. Unlike the widely accepted intuitive picture of collapse where the surface crosses the gravitational radius in the proper time, the picture of the structure and evolution of the star with asymptotically frozen layers will be reproduced in all correctly performed transitions to other frames of reference with any of their coordinates.

Under the correct such transition from the viewpoint of general relativity, it is necessary to understand those values of coordinates which describe at any moment of cosmological time on the surface of the star only those events in the internal layers that are simultaneous with the world time moment on the surface. All other descriptions based on non-simultaneous events in different parts of the star are unphysical and play only an auxiliary role for the transition to a set of simultaneous events in the entire volume of the star.

It is also important the fact that the worldlines of the star's particles in the exact solutions of the Einstein equations obtained by the OS method cover every moment of the existence of these particles in the real world and therefore these solutions give a complete picture of the evolution of the star. The irreversible dilation of the proper times due to relativistic and gravitational time dilations is the objective physical phenomenon that stops all processes in the star, including the process of collapse itself. This specific and fundamental physical phenomenon basically distinguishes the collapse scenario of the Einstein gravity from the Newtonian one, where there is no such stopping mechanism.

Thus, the collapse of the homogeneous dust star in general relativity in the exact solutions by the OS method does not lead to the black holes with a horizon on the gravitational radius and a singularity at the center, as it has been stated earlier from intuitive considerations, but at any finite moment of cosmological time leads only to the frozen star 
or the frozar, an object having almost homogeneous and practically frozen internal structure with timelike worldlines of the particles of finite mass.

\section{Appendix 1. Parametrized form of the trajectory functions}

In the literature, it is widely used a parametrized solution for the trajectories of particles of the homogeneous dust star. Thus, for convenience of comparison and further using of the above presented results, below the solutions are presented also in the such parametrized form.

\section{a. Elliptic velocities.}

For solutions outside the star let us introduce a parametrization with $a=a(t)$, $a_{0}=a(0)$ :

$$
r=a \sin \chi, \quad R=a_{0} \sin \chi, \quad a=a_{0}(1+\cos \eta) / 2, \quad f=-\sin ^{2} \chi
$$

This gives:

$$
r=\frac{R}{2}(1+\cos \eta)=R \cos ^{2}(\eta / 2), \quad F=r_{g}=R \sin ^{2} \chi
$$

and the trajectory equations (16) and (22) take the standard form (Landau \& Lifshits, 1994; Mizner, Thorne, \& Wheeler, 1973):

$$
\begin{aligned}
\tau & =\frac{R}{2 \sin \chi_{b}}(\eta+\sin \eta), \\
\frac{t}{r_{g}} & =\ln \frac{\cot \chi_{b}+\tan (\eta / 2)}{\cot \chi_{b}-\tan (\eta / 2)}+\cot \chi_{b}\left(\eta+\frac{1}{2 \sin \chi_{b}}[\eta+\sin (\eta)]\right) .
\end{aligned}
$$

Inside the star, the expressions for $f$ and $F$ have the form:

$$
f=-\frac{r_{g, R}}{R}=-r_{g} \frac{R^{2}}{R_{b}^{3}}=-\frac{\sin ^{2} \chi}{\sin ^{3} \chi_{b}}, \quad F=r_{g} \frac{R^{3}}{R_{b}^{3}}=a_{0} \frac{\sin ^{6} \chi}{\sin ^{3} \chi_{b}}
$$

In (134), instead of $r$, we insert $r_{+}$from (88) and instead of (136) we come to a more complicated expression.

b. Hyperbolic velocities.

In this case, the parametrization has the form:

$$
r=a \sinh \chi, \quad R=a_{0} \sinh \chi, \quad a=a_{0}(\cosh \eta-1) / 2, \quad f=\sinh ^{2} \chi .
$$

This gives:

$$
r=\frac{R}{2}(\cosh \eta-1)=R \sinh ^{2}(\eta / 2), \quad r_{g}=R \sinh ^{2} \chi
$$

and the trajectory functions outside the star (15) and (60) take the form:

$$
\begin{aligned}
& \tau-\tau_{0}=\frac{R}{2 \sinh \chi_{b}}(\eta-\sinh \eta), \quad \tau_{0}=-\frac{R}{\sinh \chi_{b}}\left(2^{1 / 2}-\ln \left(1+2^{1 / 2}\right)\right), \\
& \frac{t}{r_{g}}=\ln \left(\frac{\tanh (\eta / 2)+\tanh \chi}{\tanh (\eta / 2)-\tanh \chi}\right)+\operatorname{coth} \chi\left(\frac{2^{3 / 2}+\eta-\sinh \eta}{2 \sinh ^{2} \chi}-2^{3 / 2}-\eta\right) .
\end{aligned}
$$

Inside the star, the expressions for $f$ and $F$ have the form: 


$$
f=\frac{r_{g, R}}{R}=r_{g} \frac{R^{2}}{R_{b}^{3}}=\frac{\sinh ^{2} \chi}{\sinh ^{3} \chi_{b}}, \quad F=r_{g} \frac{R^{3}}{R_{b}^{3}}=a_{0} \frac{\sinh ^{6} \chi}{\sinh ^{3} \chi_{b}} .
$$

In (139) instead of $r$, we insert $r_{-}$from (88) and instead of (141) we come to a more complicated expression.

\section{Appendix 2. Derivation of the auxiliary function $y$}

a. Parabolic velocities.

A solution of the equation (78):

$$
\frac{y^{\prime}}{\dot{y}}=-\frac{\left(r_{g, R} r\right)^{1 / 2}}{R}
$$

we look for among the functions of the form

$$
y(R, r)=A(R)+B(R, r),
$$

where $A$ does not depend on $r$, and the derivative of $B$ under $R$ vanishes:

$$
y^{\prime}=A^{\prime}+B^{\prime}+\frac{\partial B}{\partial r} r^{\prime}=A^{\prime} .
$$

This gives

$$
B^{\prime}=-\frac{\partial B}{\partial r} r^{\prime}, \quad \dot{y}=\frac{\partial B}{\partial r} \dot{r} .
$$

The diagonality condition (78) then takes the form

$$
\frac{y^{\prime}}{\dot{y}}=A^{\prime}\left(\frac{\partial B}{\partial r} \dot{r}\right)^{-1}=\dot{r} r^{\prime}
$$

from which it follows:

$$
A^{\prime}=\dot{r}^{2} r^{\prime} \frac{\partial B}{\partial r}=\frac{r_{g, R}}{R} \frac{\partial B}{\partial r} .
$$

Since the left-hand side of (148) does not depend on $r$, the right-hand side must not contain $r$, which means that $B$ has the form $B=q(R) r$ and from (145) we obtain for $q$ :

$$
B^{\prime}+\frac{\partial B}{\partial r} r^{\prime}=q^{\prime} r+q r^{\prime}=0 .
$$

Taking into account $r^{\prime}=r / R$, this equation and its solution have the form:

$$
\frac{q^{\prime}}{q}=-\frac{r^{\prime}}{r}=-\frac{1}{R}, \quad \ln q=-\ln R+\ln w, \quad q=\frac{w}{R} .
$$

We find the constant $w$ from the matching on the surface. As it is clear from (52), on the surface we have $t=M\left(r / r_{g}\right)$ and $y=r / r_{g}$, and therefore, by taking into account (149), we obtain:

$$
y\left(R_{b}, r\right)=\frac{w}{R_{b}} r=\frac{r}{r_{g}}, \quad w=\frac{R_{b}}{r_{g}}, \quad q\left(R_{b}\right)=\frac{w}{R_{b}}=\frac{1}{r_{g}} .
$$

Thus, we obtain:

$$
q=\frac{w}{R}=\frac{R_{b}}{R r_{g}}, \quad B=q r=\frac{R_{b}}{R r_{g}} r .
$$

Substituting this into (147), we obtain also: 


$$
A^{\prime}=\frac{r_{g, R}}{R} q=\frac{R}{R_{b}^{2}}, \quad A=\frac{1}{2} \frac{R^{2}}{R_{b}^{2}}+p,
$$

where the constant $p$ is also found from the matching on the surface $y=r / r_{g}$ :

$$
A\left(R_{b}\right)=\frac{1}{2}+p=0, \quad p=-\frac{1}{2} .
$$

As a result, we obtain the final solution (Oppenheimer \& Snyder, 1939):

$$
\begin{gathered}
A(R)=\frac{1}{2}\left(\frac{R^{2}}{R_{b}^{2}}-1\right), \\
y(R, \tau)=\frac{1}{2}\left(\frac{R^{2}}{R_{b}^{2}}-1\right)+\frac{R_{b}}{R r_{g}} r .
\end{gathered}
$$

The derivatives of $y$ are equal to:

$$
y^{\prime}=\frac{R}{R_{b}^{2}}, \quad \dot{y}=\frac{R_{b}}{R r_{g}} \dot{r}=-\frac{R^{2}}{R_{b}^{2}} \frac{1}{\left(r_{g, R} r\right)^{1 / 2}}
$$

and the diagonality condition (142) is satisfied.

b. Hyperbolic and elliptic velocities.

In the diagonality condition (78)

$$
\frac{y^{\prime}}{\dot{y}}=\frac{\dot{r} r^{\prime}}{1 \pm r_{g, R} / R}=-\frac{\left(r_{g, R} r\right)^{1 / 2}}{R \pm r_{g, R}}(1 \pm r / R)^{1 / 2},
$$

the solution for $y$, according to the OC method [4], we search among the functions having the form (143). This gives (144) - (145) and the equation (157) then takes the form

$$
\frac{y^{\prime}}{\dot{y}}=A^{\prime}\left(\frac{\partial B}{\partial r} \dot{r}\right)^{-1}=\frac{\dot{r} r^{\prime}}{1 \pm r_{g, R} / R},
$$

from which it follows:

$$
A^{\prime}=\frac{\dot{r}^{2} r^{\prime}}{1 \pm r_{g, R} / R} \frac{\partial B}{\partial r}=\frac{r_{g, R}}{R \pm r_{g, R}}(1 \pm r / R) \frac{\partial B}{\partial r} .
$$

Since $A^{\prime}$ does not depend on $r$, the right-hand side of (159) does not depend on $r$ also, and therefore

$$
\begin{gathered}
\frac{\partial B}{\partial r}=\frac{q(R)}{1 \pm r / R}, \\
B=q \int \frac{d r}{1 \pm r / R}= \pm q R \ln (1 \pm r / R) .
\end{gathered}
$$

From (145) it follows that:$$
q^{\prime} r+q r^{\prime}=0
$$

and taking into account $r^{\prime}=r / R$ we obtain:

$$
\frac{q^{\prime}}{q}=-\frac{1}{R}, \quad \ln q=-\ln R+\ln w, \quad q=\frac{w}{R} .
$$

Then we find the constant $w$ from the matching on the surface. It is clear from (22) and (60) that on the surface $t=M\left(r / r_{g}\right)$ and $y=r / r_{g}$, which, taking into account (163), gives: 


$$
y\left(R_{b}, r\right)=\frac{w}{R_{b}} r=\frac{r}{r_{g}}, \quad w=\frac{R_{b}}{r_{g}}, \quad q\left(R_{b}\right)=\frac{w}{R_{b}}=\frac{1}{r_{g}} .
$$

Hence we have:

$$
q=\frac{w}{R}=\frac{R_{b}}{R r_{g}}, \quad B= \pm \frac{R_{b}}{r_{g}} \ln (1 \pm r / R) .
$$

Substituting this into (159), we obtain:

$$
A^{\prime}=\frac{R}{R_{b}^{2}} \frac{1}{1 \pm r_{g, R} / R}, \quad A= \pm \frac{R_{b}}{2 r_{g}} \ln \frac{1 \pm r_{g, R} / R}{1 \pm r_{g} / R_{b}} .
$$

Finally, substituting (165) and (166) in (143) we find the final solution:

$$
y(R, \tau)= \pm \frac{R_{b}}{r_{g}} \ln \frac{\left(1 \pm r_{g, R} / R\right)^{1 / 2}(1 \pm r / R)}{\left(1 \pm r_{g} / R_{b}\right)^{1 / 2}} .
$$

The derivatives of $y$ are equal to:

$$
y^{\prime}=A^{\prime}= \pm \frac{R}{R_{b}^{2}} \frac{1}{1 \pm r_{g, R} / R}, \quad \dot{y}=\frac{R^{2}}{R_{b}^{2}\left(r r_{g, R}\right)^{1 / 2}} \frac{1}{(1 \pm r / R)^{1 / 2}} .
$$

and their ratio satisfies the diagonality condition (158).

\section{References}

Datt, B. (1938). Z. Phys. A, 108, 314. doi: 10.1007/BF01374951

Friedmann, A. (1922). Z. Phys., 10, 376.

Klein, O. (1961). Ein. Prob. allgem Rel. In W. Heisenb. Phys. unz. Z. (p. 58).

Landau, L., \& Lifshits, E. (1994). Classical Theory of Fields.

Lemaître, G. (1933). Ann. Soc. Sci. Bruxells, Ser. A, 53, 51.

Mizner, C., Thorne, T., \& Wheeler, J. (1973). Gravitation.

Okun, L., Selivanov, K., \& Telegdi, V. (2000). Amer. J. Phys., 68, 115.

Oppenheimer, J., \& Snyder, H. (1939). Phys. Rev., 56, 455. doi: 10.1103/PhysRev.56.455

Schwarzschild, K. (1916). Sitz. Preuss. Akad. Wiss., 189.

Tolman R. (1934). Proc. Nat. Acad. Sci. USA, 20, 169.

Weinberg, S. (1972). Gravitation and cosmology.

Zakir Z. (2017 a). Theor. Phys., Astroph. \& Cosm., 12, 1. doi: 10.9751/TPAC.6000-043 